\title{
The ESSnuSB Accumulator
}

\author{
Elena Wildner* \\ CERN \\ E-mail: elena.wildnerecern.ch
}

The $2.0 \mathrm{GeV}, 5 \mathrm{MW}$ proton linac for the European Spallation Source, ESS, will have the capacity to send extra pulses to a neutrino target, giving an excellent opportunity to produce a high performance neutrino beam, the ESS neutrino Super Beam (ESSnuSB). The neutrino target focusing system needs pulses in the order of a few micro-seconds. These short pulses will be produced in an accumulation ring, capable of storing $2.86 \mathrm{~ms}$ long linac pulses with $1.110^{15}$ protons in each pulse. One of the main challenges of this accumulator ring is the injection of the particles. The injection method presently used in the design is H- stripping and painting. Some other ideas like lossless, multi-turn and multi-plane injection of protons will also be investigated. The evolution of the beam distribution is studied during accumulation in order to make sure that the beam properties meet the requirements for the extraction region, the transfer line and the target system. Simulation results and plans for future work on the accumulator design in view of the ESSnuSB design report, work partly funded by EU (H2020) are discussed.

The 19th International Workshop on Neutrinos from Accelerators-NUFACT2017

25-30 September, 2017

Uppsala University, Uppsala, Sweden

${ }^{*}$ Speaker. 


\section{The ESS Linac and the neutrino option}

The European Spallation Source (ESS) [1] presently being built in Lund, Sweden, will be a research centre that will have the world's most powerful neutron source, using a high current proton linac to accelerate protons to impinge a target to produce spallation neutrons. The accelerator will be a $5 \mathrm{MW}$ superconducting proton linac delivering beams of $2.0 \mathrm{GeV}$ to the target in pulses of $2.86 \mathrm{~ms}$ duration with a repetition rate of $14 \mathrm{~Hz}$. The ESS proton linac will be operational in 2023, with reduced energy and power $(1.5 \mathrm{GeV}$ and $3 \mathrm{MW})$. For the full energy more funding is needed. The layout of the ESS linac is shown in Fig. 1.

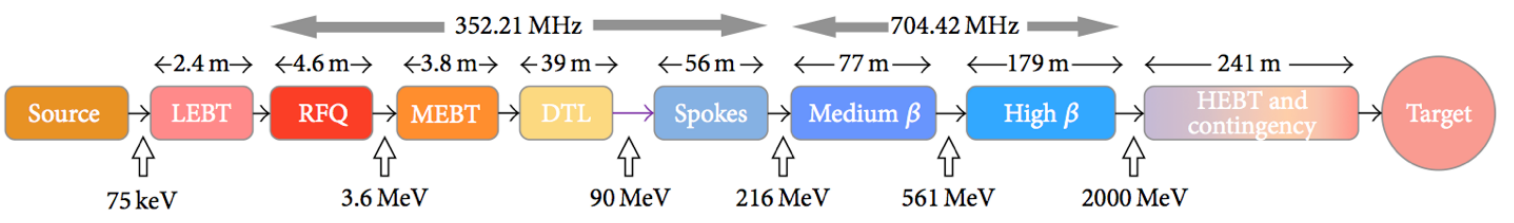

Figure 1: Layout of the ESS linac.

By pulsing the $2.0 \mathrm{GeV}$ ESS linac at $28 \mathrm{~Hz}$, twice the nominal frequency, additional interleaved $\mathrm{H}^{-}$pulses can be accelerated and sent to a neutrino production target system. This gives an excellent opportunity to produce neutrino beams with unprecedented intensity [2], the number of protons on target per year being $2.710^{23}$. The ESS linac at $28 \mathrm{~Hz}$, accelerating beams for production of both neutrons and neutrinos, will deliver $10 \mathrm{MW}$, twice the nominal beam power.

Protons impinging on a target dedicated to neutrino production will produce hadrons that need to be focused in a collector before they decay in a channel, built for the purpose, to give neutrinos. However, the Van der Meer horn type hadron collector that is presently part of the baseline for ESSnuSB, cannot handle $2.86 \mathrm{~ms}$ long linac pulses due to ohmic heating of the magnet system current leads. Therefore, the linac pulse has to be accumulated in a storage ring to produce shorter pulses of only a few $\mu$ s duration, before being sent to the neutrino production target. The design of such an accumulator, will have impact on the ESS linac. The injection of the beam from the linac into the accumulator is performed by injecting more than 500 turns letting, at the same time, the injected beam circulate in the accumulator (multiturn injection). To make the injection process efficient, $\mathrm{H}^{-}$ions are accelerated in the linac and the protons are produced by stripping the $\mathrm{H}^{-}$ions in a foil at the injection point. Other stripping methods, like laser stripping, are under development at other laboratories and may be considered for the ESSnuSB accumulator for future upgrades. To accelerate $\mathrm{H}^{-}$ions, an $\mathrm{H}^{-}$source needs to be added to the ESS linac, including extra LEBT, RFQ and MEBT sections for matching both the $\mathrm{H}^{-}$beam and the proton beams.

The additional $5 \mathrm{MW}$ beam to be accelerated for the production of neutrinos and the different linac pulsing schemes needed to mitigate negative effects related to the high intensities in the accumulator, will have implications for the different accelerator hardware systems. In particular, the cooling water system and the cryogenic distribution and capacity, the radio frequency couplers, the klystron modulators, the electric transformers, and the power capacity, have been subject to preliminary studies [5]. These studies do not reveal show stoppers for the additional beam power in the linac, but radiation aspects need dedicated studies for the $\mathrm{H}^{-}$option. In addition, less than 


\begin{tabular}{cccc}
\hline Parameter & Value (n) & Upgrade $\mathbf{n}+v$ & Upgrade $\mathbf{n}+v$ \\
\hline Average beam power & $5 \mathrm{MW}$ & $10 \mathrm{MW}$ & $10 \mathrm{MW}$ \\
\hline Ion kinetic energy & $2 \mathrm{GeV}$ & $2 \mathrm{GeV}$ & $2.5 \mathrm{GeV}$ \\
\hline Average macro pulse current & $62.5 \mathrm{~mA}$ & $62.5 \mathrm{~mA}$ & $50 \mathrm{~mA}$ \\
\hline Average macro pulse length & $2.86 \mathrm{~ms}$ & $>2.86 / 4 \mathrm{~ms}$ & $>2.86 / 4 \mathrm{~ms}$ \\
\hline Pulse repetition rate & $14 \mathrm{~Hz}$ & $>28 \mathrm{~Hz}$ & $>28 \mathrm{~Hz}$ \\
\hline Linac length & $352.5 \mathrm{~m}$ & $352.5 \mathrm{~m}$ & $352.5+\mathrm{ca} 70 \mathrm{~m}$ \\
\hline
\end{tabular}

Table 1: ESS Linac Paramemters

$100 \mathrm{~ns}$ gaps in the linac beam, at a frequency corresponding to the accumulator circumference, are needed to be able to operate the extraction kickers in the accumulator in order to achieve minimal particle loss at beam extraction. These gaps may create Higher Order Modes (HOM) in the superconducting cavities.

The requirements for maintenance and machine protection set a strict limit on beam losses. For high power linacs this is a concern and therefore it is crucial to design a linac which does not excite particles to beam halo and that keeps the emittance growth to a minimum to avoid losing particles. The ESS linac is designed carefully to minimise such effects all along the linac and the transfer lines. To accelerate another $5 \mathrm{MW}$ beam in the linac, in particular an $\mathrm{H}^{-}$beam, is demanding for the design studies.

\section{High intensity in the Accumulator}

The very high intensities to be stored may cause tune spread and tune shifts. A first estimation of these effects can be given by the Laslett tune shift formula (eq. 2.1)

$$
\Delta Q_{x, y}=-\frac{r_{0} N}{2 \pi E_{x, y} \beta^{2} \gamma^{3} B f}
$$

where $r_{0}$ is the proton radius, $N$ the intensity, $E$ the emittance, $\beta^{2} \gamma^{3}$ the relativistic factors and $B f$ the bunching factor.

There are two proposals to mitigate the problems due to the high intensity in the accumulator. The $2.86 \mathrm{~ms}$ linac beam can be injected into several accumulators using a beam distributer at injection. Alternatively the linac beam may be produced in several pieces of similar intensity but only $2.86 / \mathrm{n} \mathrm{ms} \mathrm{long,} \mathrm{to} \mathrm{be} \mathrm{injected} \mathrm{one} \mathrm{after} \mathrm{the} \mathrm{other} \mathrm{into} \mathrm{one} \mathrm{accumulator} \mathrm{and} \mathrm{extracted}$ immediately after complete injection of shorter beam, $\mathrm{n}$ being the number of shorter $\mathrm{H}^{-}$beams accelerated by the linac.

The required emittance and the distribution of the particles in the beam can be adjusted by the painting process, by optimising the extraction beam line up to the target, and by adequate target requirements. The relativistic properties of the beam from the accumulator are set by the physics goal of the facility and the linac energy. The proton beam will not be accelerated in the accumulator ring (actual base-line design), so the energy will be set by the linac energy. The bunching factor, $B f$ in formula 2.1 is at present taking into account the $10 \%$ beam gap for extraction, but not any influence of the longitudinal micro-structure in the beam from the linac rf. Simulations will refine this assumption. The accumulator must have a reasonable footprint on the ESS site, and to be able to hold $1.110^{15}$ particles. Multiple accumulators with a beam distribution system at injection can 
be envisaged. Another possibility is to produce a number of shorter linac beam pulses, accumulate and extract these shorter beams, one after the other, sequentially. The total number of particles accelerated and accumulated would be the same but the accumulator would contain only a fraction of the total intensity for each short beam pulse from the linac. The lattice will be similar for the multiple ring case and for the case where shorter linac pulses are injected. The injection painting and the injection optics have to be adapted due to foil heating which will become more important for the one ring case, since all $1.110^{15}$ particles need to be stripped by the same foil during the shorter $71.4 \mathrm{~ms}$, corresponding to $14 \mathrm{~Hz}$. This means that the beam size on the foil will need to be adapted to keep the foil temperature within limits set by the foil material and the foil design.

The different options proposed to reduce the high intensity issues in the accumulator are illustrated in Fig. 2. The upper part of the figure shows the $28 \mathrm{~Hz}$ pulsing of the linac with one proton (blue) and one $\mathrm{H}^{-}$(green) pulse interleaved. To reduce the intensity in the accumulator, the $\mathrm{H}^{-}$ pulse is split between several vertically stacked accumulator rings. The brown curve shows the rf power. The width of the pulses represents the duration of the linac beam pulse. The second part from the top shows three pulses inserted between the neutron production pulses, each one having a duration of one third of the total linac beam pulse duration, i.e. $2.86 / 3 \mathrm{~ms}$. The pulses have the same intensity as the nominal linac beam pulses and the overhead to fill the cavities will appear 3 times. The third case shown at the bottom of Fig. 2 is interesting due to the fact that the overhead in rf power to fill the cavities only appears once for one $2.86 \mathrm{~ms}$ long $\mathrm{H}^{-}$pulse from the linac, and is therefore not different from the $28 \mathrm{~Hz}$ case. The reason is that two small gaps in the linac beam of at least $100 \mu \mathrm{s}$ would, for this third case, permit the the painting bumpers to re-arm and be ready to paint the following two $2.86 / 3 \mathrm{~ms}$ long parts of the $\mathrm{H}^{-}$linac pulse. Comparing with the CERN PS Booster distributer system for injection, the maximum allowed gap size of $100 \mu$ s would allow a feasible injection painting system for the ESSnuSB accumulator [3]. The drift tube linac and the klystrons could probably, with today's hardware, be operated at a maximum of $10 \% \mathrm{rf} \mathrm{DC}$, the RFQ may even have a lower limit. The $28 \mathrm{~Hz}$ case yields an rf duty cycle of $8.4 \%$ and $56 \mathrm{~Hz}$ yields an rf duty cycle of $9.45 \%$. See [5].

The target station requirements must be such that the collector focusing can handle the pulse sequence from the accumulator. In the multi ring case, $28 \mathrm{~Hz}$ linac pulsing, the timing of the beam extraction from the rings can be adapted, however in this case the beams are circulating in the various rings at different time intervals which may give beams from the accumulator rings having different beam quality. In the second case of Fig. $2(56 \mathrm{~Hz})$ the arrival of the beam from the accumulator is fixed by the $56 \mathrm{~Hz}$ linac pulsing. Equally so in the bottom case $(28 \mathrm{~Hz})$, however for this option the target must be able to accept beams with intervals corresponding to $2.86 / 3 \mathrm{~ms}$, which is the time needed to accumulate $1 / 3$ of the linac beam in the ring.

\section{Accumulator Lattice Design}

Following the design concept of the SNS lattice, where the magnet fields in the original 1 $\mathrm{GeV}$ lattice are rather moderate, a first lattice is considered, keeping the $30 \mathrm{~m}$ injection straight sections unchanged for $2 \mathrm{GeV}$. However, in view of the large apertures required, due to large beam sizes, it makes sense to keep the bending fields in the arcs as conservative as in the SNS; hence the arc length was doubled. The circumference is increased from 248 to $376 \mathrm{~m}$, reducing the 


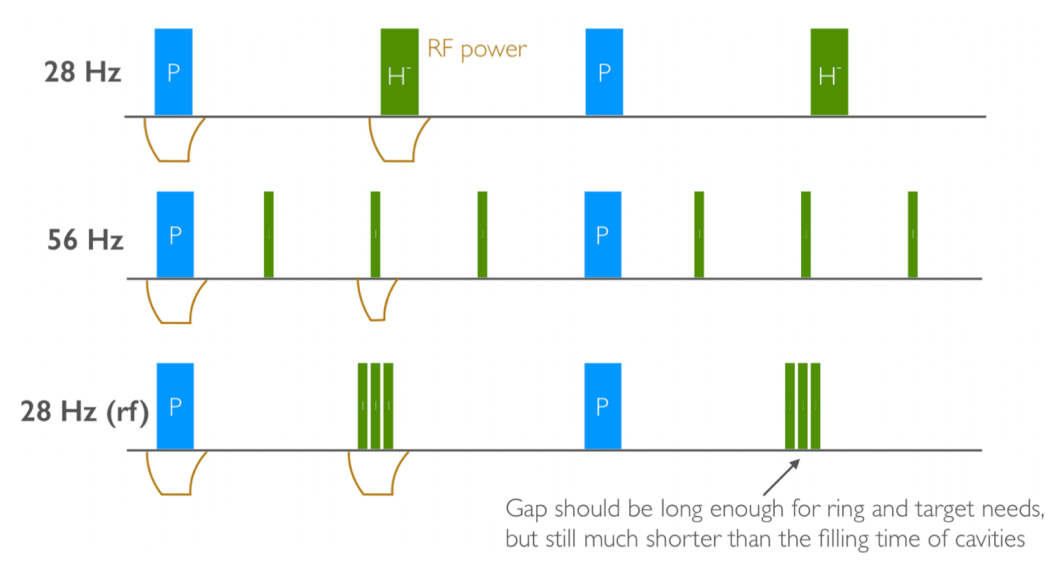

Figure 2: Pulse distribution for the the 3-ring and the one-ring option: Three stacked accumulators can accumulate one third of a linac pulse in each ring to send the total intensity to the target (upper) or one accumulator can send three consecutive pulses to the target (the two lower configurations), see text.

number of turns injected per fill. The collimation layout (in the first long straight section after the injection section) remains the same [4]. Using this lattice for $1 / 4$ of the total intensity gave a tune spread less than 0.2 which is considered within the tolerable limit [8]. Additional simulations, also using $1 / 4$ of the total intensity [6], with multi-turn injection and anti-correlated painting bumps using a J-PARC like lattice show acceptable space charge at $2 \mathrm{GeV}$. A simple lattice design and the use of SNS like magnets would, according to these simulations, be suitable for the ESSnuSB application. The first assumptions of intensity in the accumulator ring was 1/4 of the total intensity, however, as mentioned, $1 / 3$ of the intensity would be a better option to have fewer vertically stacked accumulators or lower pulsing rates of the linac [5]. A lattice version with shorter arcs, has been developed at CERN and is now under test.

To accelerate and inject protons would be a significant simplification not only of the injection equipment but also result in a smaller beam loss in the linac and a simpler linac design at lower energies. No additional $\mathrm{H}^{-}$source would be needed. The injection preserves volumes in phase space by using a tilted electrostatic septum and correlated painting with orbit bumps in the transverse plane to avoid beam loss at the septum [7]. No existing operational application exists yet, however, the Chinese High Intensity Heavy Ion Accelerator Facility, HIAF, to be built in Huizhou, will use the tilted septum injection scheme. Applied to a J-PARC like ring with ESS parameters would, for 550 turns, give no beam loss according to simulations (without space charge). $100 \mathrm{~mm}$ mrad emittance, as required for the current extraction line design may be too constraining. Again, the intensity for these simulations is $1 / 4$ of the total required.

In Fig. 3 the temperature of the foil has been calculated for the case of 3 sequencial linac batches with a preliminary lattice [8]. The foil temperature for this simulation will reach $1828 \mathrm{~K}$ for a carbon foil (emittance $75 \mathrm{~mm}$ mrad geometric at $3 \sigma$ ). The linac emittance is $0.33 \mathrm{~mm} \mathrm{mrad}$ for this simulation (linac nominal emittance is $0.25 \mathrm{~mm}$ mrad). An experimentally acceptable value for the foil temperature is $2040 \mathrm{~K}$ (carbon melting temperature is $3773 \mathrm{~K}$ ), see [9]. The simulation shows the feasibility of using a carbon foil. The foil temperature can be optimised by changing the injection optics, linac emittance or injection painting. 
The work on the accumulator, will be continued during the next four years with funding recently obtained from the EU H2020-INFRADEV-1-2014-1 call, Proposal number: 653428, the acronym of the project being ESSnuSB.

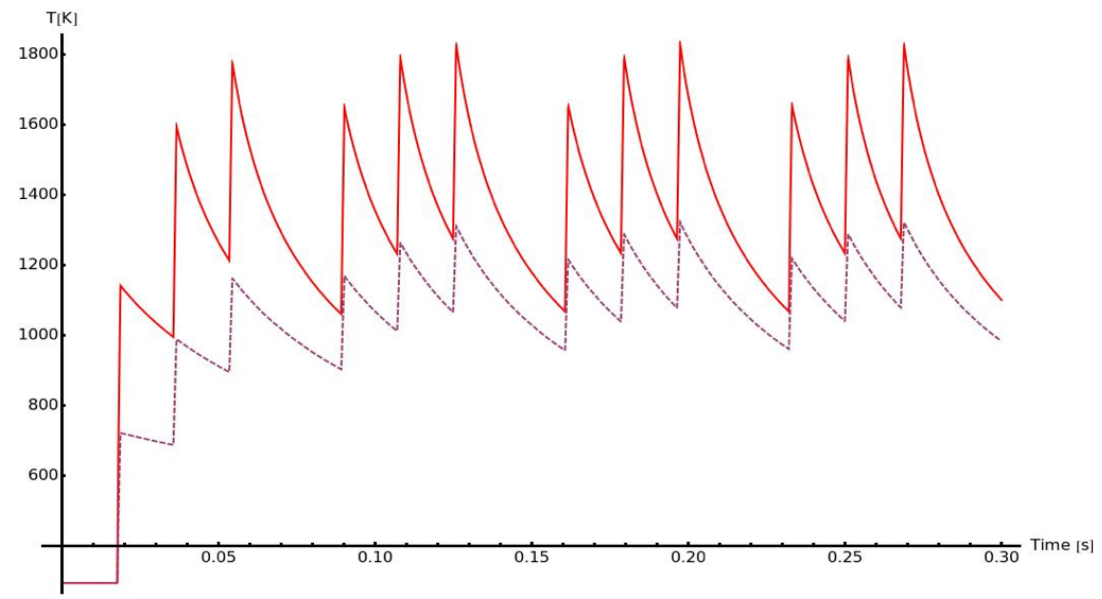

Figure 3: Evolution of maximum foil temperatures $[\mathrm{K}]$ at the $\mathrm{H}^{-}$- spot peak (dashed) and the combined $\mathrm{H}^{-}$and circulating protons peak (red) for 3 injections into the accumulator per linac pulse. The temperature reaches $1828 \mathrm{~K}$.

No more than $1 \mathrm{~W} / \mathrm{m}$ beam loss allowed on the tunnel walls, operation with an average beam power of $5 \mathrm{MW}$ would require a maximal fractional loss of $0.210^{-6}$. For the circumference of the accumulator ring this corresponds to a fractional loss of in the order of $10^{-4}$. For the transfer lines the allowed losses have been limited to $0.1 \mathrm{~W} / \mathrm{m}$. Taking this into account, the Lorentz stripping of the $\mathrm{H}^{-}$ions in the transfer lines imposes an upper limit on the curvature of the beam lines, 0.15 $\mathrm{T}$ and $2.5 \mathrm{GeV}$ give $\mathrm{B} \rho 11.02 \mathrm{Tm}$ where $\rho=73.5 \mathrm{~m}$ in the dipoles. Assuming $66 \%$ dipole filling factor, gives a bending radius of the transfer line tunnel of $111 \mathrm{~m}$. This value is used for the design of the foot-print of the ESS Accumulator on the ESS site.

\section{References}

[1] ISBN 978-91-980173-2-8

[2] Advances in High Energy Physics, vol. 2016, pp. , 2016.

[3] B. Goddard, private communication

[4] SNS Design Manual 2003

[5] F. Gerigk and E. Montesinos, CERN-ACC-NOTE-2016-0050

[6] S. Machida, https://indico.esss.lu.se/event/618/session/3/contribution/1

[7] C.Prior, Studies of High Intensity Proton FFAGs at RAL, HB2016-Proceedings, Malmö, 2016

[8] Olvegård et al., Overview of the ESSnuSB Accumulator Ring, HB2016-Proceedings, Malmö, 2016

[9] Liaw et al., PAC N.Y. 1999, pp 3300 